\title{
Les causes de la mortalité pendant la Grande
}

\section{Famine}

The Causes of mortality during the Great Famine

\section{Philippe Brillet}

\section{(2) OpenEdition}

\section{Journals}

\section{Édition électronique}

URL : http://journals.openedition.org/rfcb/228

DOI : $10.4000 /$ rfcb. 228

ISSN : 2429-4373

Éditeur

CRECIB - Centre de recherche et d'études en civilisation britannique

\section{Édition imprimée}

Date de publication : 1 septembre 2014

Pagination : $33-49$

ISSN : 0248-9015

\section{Référence électronique}

Philippe Brillet, "Les causes de la mortalité pendant la Grande Famine », Revue Française de Civilisation Britannique [En ligne], XIX-2 | 2014, mis en ligne le 01 mai 2015, consulté le 30 avril 2019. URL : http:// journals.openedition.org/rfcb/228; DOI : 10.4000/rfcb.228

\section{(c) (i) $\odot$}

Revue française de civilisation britannique est mis à disposition selon les termes de la licence Creative Commons Attribution - Pas d'Utilisation Commerciale - Pas de Modification 4.0 International. 


\title{
Les causes de la mortalité pendant la Grande Famine
}

\author{
Philippe BRILLET \\ Université Toulouse 2 - Jean Jaurès
}

La question des causes de la mortalité pendant la Grande Famine est assez délicate. S'il apparaîtra, j'espère, d'emblée évident que « mourir de faim » ne saurait être davantage qu'un terme générique recouvrant de multiples facteurs, nutritionnels bien sûr mais aussi infectieux ou digestifs, pour ne citer que les principaux, explorer ici la dimension clinique et la morbi-mortalité de la Famine n'est pas sans difficulté. D'abord parce que la physiopathologie humaine n'est pas un prérequis de l'agrégation d'anglais, ensuite parce que la combinaison de ces facteurs fait encore l'objet de débats parmi les spécialistes.

Il ne s'agira donc pas dans cet article de faire un point, fût-il partiel, de l'état des connaissances médicales, mais d'éclairer un fait complexe de civilisation en présentant un certain nombre de données et de concepts qui semblent d'autant plus susceptibles de compléter l'intelligence de ce fait qu'ils sont rarement exposés. Mais l'ampleur même de ce projet explicatif, qui devra examiner les systèmes agroalimentaires avant de passer à la physiopathologie humaine et à la santé publique, implique de se limiter à la seule échelle de l'Irlande entière. Ceci est d'autant plus nécessaire que, comme on le verra, la mauvaise qualité des statistiques de mortalité rend très aléatoire l'élaboration de ce qui serait une géographie de la mortalité au cours de la Famine. Elle n'est aujourd'hui, malgré de nombreux efforts, qu'un work in progress ${ }^{1}$. Notons au passage que la géographie de la réponse médicoadministrative est un peu mieux connue, ce qui est d'ailleurs inhabituel, grâce au travail de recherche et de synthèse de plusieurs auteurs basés à Cork, notamment Laurence M. Geary ${ }^{2}$.

S'agissant donc des causes de la mortalité à l'échelle de l'Irlande entière, la toute première question à examiner est celle de l'état de santé des Irlandais à la veille de la Famine, car cet état conditionnait leurs capacités de résistance, d'autant que le fléau n'allait pas durer moins de six années et, ainsi, user jusqu'à l'extrême sa population-cible qui sera frappée de façon cumulative. Ceci nous conduira d'abord à examiner le système nutritionnel tel qu'il existait en 1845, et comment il s'était mis en place sur des sols réputés peu favorisés. Nous envisagerons ensuite la morbi-

\footnotetext{
${ }^{1}$ Pour une présentation de son état des lieux, on pourra se reporter au chapitre du même auteur au sein de l'ouvrage sur la Famine en cours d'impression aux Presses universitaires de Rennes.

${ }^{2}$ On pourra se référer à Laurence M. GEARY, « The medical profession, health care and the poor law in nineteenth-century Ireland », pp. 189-206 in Virginia CROSSMAN et Peter GRAY (eds), Poverty and Welfare in Ireland, 1838-1948, Dublin : Irish Academic Press, 2011.
} 
mortalité proprement dite, d'abord en essayant de préciser la débilitation induite par la faim chronique, puis en étudiant les diverses infections qui ont été à l'œuvre, avant d'examiner enfin - et de façon plus brève - les insuffisances théoriques des praticiens de l'époque.

\section{A la veille de la Famine, des Irlandais finalement plutôt bien nourris?}

Les opinions sur ce point apparaissent pour le moins divergentes. Pour certains, tel l'historien français Pierre Joannon ${ }^{3}$, la santé des Irlandais était tout simplement effroyable: "En 1824, un comité d'enquête de la Chambre des communes attirait l'attention du gouvernement sur «la détresse épouvantable de la population... sur les maladies contagieuses qu'elle répandait autour d'elle, sur le chiffre considérable de ceux qui étaient littéralement morts de faim.» Tel est également l'avis de Ciaran Ó Murchadha, qui ouvre son livre The Great Famine Ireland's Agony $1845-1852^{4}$ par cette première phrase :

In the mid-nineteenth century, Ireland was proverbial all over the western world for the extraordinary poverty of its people. 'Misery, naked and famishing' remarked the French traveller Gustave de Beaumont in 1848, 'that misery which is vagrant, idle, and mendicants covers the entire country'.

Mais d'autres auteurs, comme Christine Kinealy, directrice depuis 2013 du nouvel institut de recherche sur la Grande Famine à l'université américaine de Quinnipiac, sont d'une opinion toute différente, et quasi opposée :

The relationships between population, poverty and potatoes superficially was an attractive one, but was greatly overstated. However, one consequence of the high dependence on the potato of a large section of the Irish people was that on the eve of the Famine, Ireland had one of the tallest, healthiest and most fertile populations in Europe. $^{5}$

Cormac Ó Gráda, professeur d'histoire économique à University College Dublin, arrive à une conclusion assez similaire dans son ouvrage Ireland before and after the Famine ${ }^{6}$, en examinant les quelques données quantitatives disponibles: «However, if the Irish were abysmally poor by standard criteria, other evidence suggests that the picture of pre-Famine poverty based on such criteria alone is somewhat oversimplified. Not all was gloom and doom ${ }^{7}$. " Ainsi, en rétro projetant les résultats du recensement de 1841 - corrigés de l'émigration probable, il a pu calculer qu'à la veille de la Famine l'espérance de vie à un an - soit après le pic de mortalité de la première année de vie - était d'environ 50 ans, soit la moyenne

${ }^{3}$ Histoire de l'Irlande et des Irlandais, Paris : Perrin, 2009, p. 307.

${ }^{4}$ London: Bloomsbury, 2011, p. 1.

${ }^{5}$ A Death-Dealing Famine: The Great Hunger in Ireland, London: Pluto, 1997, p. 5.

${ }^{6}$ Manchester: University Press, 1993.

${ }^{7}$ p. 17. 
européenne de l'époque telle que l'on peut l'estimer. Par ailleurs, son analyse statistique de la taille des marins en service dans la Navy en 1853 et 1854 montre que les Irlandais étaient légèrement plus grands que les autres, et donc a priori un peu mieux nourris. Le même auteur obtient de plus un résultat semblable pour les régiments de l'East India Company à l'époque des campagnes napoléoniennes. Ceci est d'autant plus intéressant que les données en cause sont donc un peu plus anciennes : la taille des jeunes en âge de s'engager, et donc la ration calorique moyenne qu'ils avaient reçue depuis l'enfance, était ainsi déjà légèrement supérieure en Irlande au début du XIX ${ }^{\mathrm{e}}$. De plus, les effroyables conditions de recrutement de la Compagnie nous garantissent que ces engagés appartenaient en grande majorité, voire en quasi totalité, aux couches les plus pauvres et moins bien nourries de la population.

Bien entendu, une si grande divergence entre les auteurs n'est pas sans poser question. Une première explication potentielle réside dans les écarts entre projets politiques, et c'est là tout l'enjeu du débat historiographique ${ }^{8}$, dont on connaît l'importance majeure pour la Famine. Il ne s'agit pas ici d'entrer dans ce débat, ni de classer les quatre auteurs cités dans l'une des écoles de pensée existantes mais, d'abord, de noter le manque surprenant de consensus pour un domaine qui relève, ou devrait relever, davantage du fait que de l'interprétation et, ensuite, d'attirer l'attention sur le danger à construire sa perception globale de la Famine à partir d'un seul ouvrage pris comme référence et simplement complété par des lectures ultérieures.

Dans le cas précis de l'évaluation de l'état nutritionnel des Irlandais, les divergences sont d'autant plus compréhensibles que l'état statistique de l'Irlande d'avant la Famine ne peut être qualifié, au mieux, que de très incomplet. De plus l'état nutritionnel, en économie de subsistance, est susceptible de grandes variations tant dans l'espace (y compris entre villages voisins) que dans le temps (y compris entre les saisons d'une même année). Chaque thèse peut donc s'appuyer sur des études de cas. Toutefois, cette composante politique est loin d'être une explication satisfaisante à elle seule, ne serait-ce que parce que l'historiographie de la Famine, à

${ }^{8}$ Ce qui est traditionnellement qualifié de visée nationaliste oscillait entre deux pôles : insister sur la pauvreté, fruit de l'oppression britannique, ou sur la résilience, signe de la vitalité irlandaise. A partir des années 1930 les historiens irlandais, comme leurs collègues dans les autres pays européens, commencèrent à organiser leur discipline sur des bases plus scientifiques. Toutefois, la convergence de ce tournant avec la naissance de l'État Libre, soucieux de respectabilité en suivant le modèle britannique, dans un contexte de victoire où la rhétorique nationaliste - et ses indéniables excès - était devenue perçue comme embarrassante impliqua une remise en cause radicale - et de surcroît affichée - de ce qui devenait perçu comme des mythes. De façon assez similaire, le renouveau méthodologique général que connut l'histoire à partir des années 1960 fut, en Irlande, surtout utilisé pour prolonger ce travail d'épuration. Ce courant de pensée, qualifié de "révisionniste », insista notamment sur le caractère éminemment instable de l'Irlande rurale de 1845 , où la conjonction d'une surpopulation, d'une véritable arriération économique et d'une mauvaise gestion ne pouvait que mener à une crise de subsistance majeure démontrant de façon éclatante la pertinence de la pensée de Malthus, dont l'Essai sur les principes de population avait été publié en 1798. De fait, la façon dont Malthus est cité et mobilisé suffit bien souvent à déterminer la visée d'un ouvrage ou d'un article. 
défaut d'un véritable consensus, est maintenant apaisée ${ }^{9}$. Il s'agit en effet plutôt aujourd'hui de compenser ce qui est perçu, avec le recul, davantage comme un effacement relatif de cet épisode dans le récit de l'histoire nationale, derrière d'autres tels que les Plantations ou la Guerre d'indépendance, que comme un traitement qui aurait été partial. De plus, les données anthropo-économiques très franchement positives, présentées plus haut, apparaissent difficilement contournables, d'autant qu'elles ont été construites à partir de séries de plusieurs milliers de cas.

Ces données nous suggèrent alors une autre hypothèse : la coexistence d'une (très) grande pauvreté, laquelle fait de toute façon consensus, et d'un état nutritionnel pourtant globalement satisfaisant, malgré donc des opinions contraires de certains commentateurs et historiens. Une telle coexistence apparait sans doute étonnante, vue de la France du début du Xxi ${ }^{e}$ siècle, mais elle est encore assez répandue dans ce que l'on appelait il y a encore peu le Tiers Monde, et ne se résume donc pas aux multiples qualités de la pomme de terre per se, même si elle prend bien sûr appui sur ce tubercule. On trouve, par exemple, une situation très proche, encore aujourd'hui, dans l'essentiel du Nordeste brésilien. Les conditions climatiques y sont certes différentes de celles de l'Irlande en général, puisqu'il s'agit du «polygone de la sécheresse ${ }^{10}$, et la ration alimentaire y est fondée sur une combinaison de haricot et de mil et non sur Solanum tuberosum, mais le système final est très semblable par sa combinaison de robustesse habituelle et de vulnérabilité épisodique. C'est cette combinaison que nous allons maintenant examiner.

\section{Un système vivrier fondamentalement robuste, mais vulnérable aux accidents?}

En 1946 le géographe brésilien Josué de Castro publia la première édition de sa Géographie de la Faim ${ }^{11}$, où il présentait une typologie régionale de la faim pour son pays. L'un des grands intérêts de ce travail pionnier était de s'affranchir, pour la première fois, du classement jusque-là strictement quantitatif des états de malnutrition. De fait ce classement n'est guère utilisable, même par les ONG d'aujourd'hui, car il impose de connaître la ration alimentaire totale, l'apport énergétique global, la quantité de protéines ingérées (et notamment des huit aminoacides qui ne sont pas synthétisable par l'homme, plus un neuvième chez l'enfant), les apports en lipides, en glucides (ou sucres), en iode, en calcium, en fer et en vitamines, pour ne citer que les principaux. Tout ceci doit bien sûr être de surcroît

\footnotetext{
${ }^{9}$ Le très volumineux Atlas of the Great Irish Famine, publié conjointement en 2013 par les presses de University College Cork et New York University Press, se veut ainsi une véritable encyclopédie fondée sur de multiples données à l'échelle la plus fine.

${ }^{10} \mathrm{Il}$ importe sans doute ici de rappeler la grande diversité des régions d'Irlande en matière de pluviométrie. Si l'essentiel du littoral occidental reçoit plus de $2000 \mathrm{~mm}$ de pluie par an en moyenne, soit presque autant que la ville de Belém en Amazonie, pour rester dans la comparaison avec le Brésil, sa moitié orientale (sauf en ses extrémités nord et sud) n'en reçoit pas 800 , ce qui est précisément le cas d'une partie du polygone de la sécheresse. Notons que recevoir moins de $600 \mathrm{~mm}$ de pluie par an caractérise les régions non pas sèches, mais arides.

${ }^{11}$ Geografia da Fome, Sao Paulo: Gryphus, 1946, 361 p. Plusieurs traductions françaises sont disponibles, la plus abordable étant celle de 1972 publiée au Seuil dans la collection Politis.
} 
modulé par l'âge, le sexe, l'effort physique moyen et, pour la femme, un éventuel état de gravidité (la grossesse). Josué de Castro considéra d'abord que distinguer des zones de malnutrition chronique, également qualifiées de zones de disette, et des zones de faim endémique ${ }^{12}$ était bien suffisant, tant pour décrire la réalité que pour organiser lutte et prévention ${ }^{13}$. Il mit ensuite en évidence un troisième type de territoires, marqué par ce qu'il qualifia de «faim épidémique ». Ces terres, très différentes des précédentes et jusque-là mal identifiées, sont pour lui marquées par un contraste étonnant entre la bonne, voire très bonne, alimentation qui est celle de ses habitants la plupart du temps et la survenue, plus ou moins régulière, de graves épidémies de famine, en règle extrêmement violentes et destructrices. Je le cite :

Il s'agit là d'un type de faim complètement différent des autres. Il ne s'agit plus d'une faim à la fois permanente et souvent modérée, conséquence et expression des conditions de vie, mais d'un surgissement épidémique qui vient rompre la relative abondance qui caractérise la vie des gens la plupart du temps. [...] En temps normal, l'alimentation des gens est tout à la fois très saine sur le plan qualitatif et bien suffisante en quantité. C'est là un excellent exemple de la capacité humaine à tirer tout ce qui est lui est nécessaire d'un environnement pourtant guère privilégié. ${ }^{14}$

On pourra objecter ici que ces considérations sur le Brésil ne semblent avoir qu'une parenté lointaine avec les événements d'Irlande et qu'une transposition y serait a priori problématique, et ce pour au moins deux raisons : d'abord si l'on veut bien admettre que l'état nutritionnel des Irlandais était moins effroyable que sa caricature habituelle, voire même qu'il était sans doute décent, ne serait-il pas un peu excessif de le qualifier de bon? Ensuite, l'épisode unique de la Grande Famine ne semble guère correspondre à ces événements récurrents que furent (et sont toujours) les famines du Nordeste.

Je commence par ce second point, car il peut être établi plus rapidement. Si, de fait, une seule de ses famines est restée dans les mémoires comme «La Grande », l'Irlande a connu de multiples épisodes de disette, la toute première qui soit partiellement imputable à la pomme de terre survenant même dès l'hiver 1727-1728. Les commissaires du recensement de 1851 n'en listèrent pas moins de 24, certes de degré et d'amplitude variables, mais toujours capables de tuer sur un large territoire. Ainsi la récolte de 1739 fut-elle totalement perdue, comme celle de 1783. Mais la pomme de terre n'était encore qu'un aliment d'appoint, et ses conséquences ne furent majeures que pendant la seule période de soudure entre les récoltes de céréales, qui connaissaient leurs propres aléas. La pomme de terre permit même, dans certains cas et jusqu'à un certain point, de franchir une période difficile lorsque la récolte de céréales était déficiente. C'est le recul des emblavures ${ }^{15}$ à son profit et

${ }^{12}$ Endémique cumule ici ses deux sens : biologique (qui est spécifique d'un territoire) et médical (présence constante d'une maladie ou d'un phénomène morbide dans une région).

${ }^{13}$ Il discuta également, sans pouvoir trancher formellement, de la possible coïncidence de cette frontière entre malnutrition simple et véritable faim endémique avec celle qui sépare la grande pauvreté de la misère.

${ }_{15}^{14}$ p. 175 , ma traduction.

${ }^{15}$ Ensemencement par des graines de céréales. 
la rapide croissance démographique du premier $\mathrm{XIX}^{\mathrm{e}}$ siècle augmenta sensiblement la dépendance à son égard. Or si le climat équilibré et bien arrosé de l'Irlande convient globalement à ce tubercule, il est sensible aux périodes prolongées d'humidité, comme de sécheresse d'ailleurs, et nécessite une température minimale de huit degrés à la plantation. Il en résulta de fréquents épisodes de défaillance partielle, parfois sur de larges étendues, lorsque les conditions climatiques étaient contraires. De plus la pomme de terre se révéla également très sensible à de très nombreux agents infectieux, bactériens, viraux ou mycotiques (champignons), qui n'étaient bien sûr pas - ou très mal - connus à l'époque. L'extension constante du territoire planté, sur des sols de plus en plus pauvres et donc sources de fragilité des tubercules par carence en phosphate, fit le lit de telles contagions. Ainsi au cours de la seule décennie qui précéda la Grande Famine ${ }^{16}$, l'Irlande ne connut pas moins de cinq récoltes largement ou totalement perdues : 1836, 1837, 1839, 1841 et 1844. Il serait donc erroné de penser que l'épisode majeur qui nous préoccupe soit survenu comme un coup de tonnerre dans un ciel bleu, prenant tout le monde par surprise. Le risque de défaillance, partielle ou totale, de la récolte était bien connu. C'est son ampleur, sa durée, et la survenue d'un agent pathogène particulièrement agressif - et jusque-là inconnu en Irlande, qui prirent tout le monde de court, mais non sa survenue. Il semble que l'inquiétude fut relativement limitée en 1845, malgré l'ampleur des dommages, car nul n'imaginait une répétition immédiate : la question étant de savoir comment tenir jusqu'à la récolte de l'année suivante. On pourrait encore ajouter que la famine de 1740-41 (Bliain an Áir, «the Year of Slaughter ») est considérée par certains comme proportionnellement plus grave. La chose est possible mais ne saurait être démontrée, en raison de l'absence quasi-complète de données disponibles, et la plupart des auteurs se gardent bien de toute estimation quantitative $\mathrm{e}^{17}$.

La Grande Famine peut donc apparaître comme une sorte de primus inter pares au sein des nombreuses disettes qui ont frappé l'Irlande au cours du temps. Toutefois une telle affirmation semble rendre plus délicate encore la justification d'une alimentation de ses habitants qui serait très satisfaisante en dehors de ces crises. Pour le Nordeste, de Castro l'expliquait par la combinaison de deux facteurs principaux $^{18}$ :

- une alimentation de qualité : "L'essentiel de l'énergie est fournie par le mil, alors que les vitamines et les amino-acides viennent du lait. Ce dernier n'est, nulle part ailleurs dans ce pays et même dans les régions les plus prospères, aussi disponible pour les pauvres. »

- l'absence de toute exportation, l'agriculture étant strictement vivrière : «Les habitants ne sont pas des fermiers travaillant pour les marchés étrangers et à la

\footnotetext{
${ }^{16}$ Le Nordeste connut parallèlement en 1877-1879 ce qui reste dans l'histoire comme la Grande Sécheresse, parce qu'elle fut de loin la plus effroyable, mais cet épisode ne fut qu'un parmi beaucoup d'autres.

${ }^{17}$ Leslie A. CLARKSON et E. Margaret CRAWFORD, dans un article intitulé «A NonFamine History of Ireland » (History Ireland, Vol. 10, Issue 2, Summer 2002, pp. 31-35), se risquent pourtant à proposer le chiffre de 480000 morts.

${ }^{18}$ Pour cet auteur deux autres facteurs interviennent également : un respect marqué pour le sol, que l'on sait fragile en pays de sécheresse et un recours occasionnel au nomadisme pour diminuer la pression sur eux. Ils n'ont certes guère d'équivalence en Irlande, mais de Castro ne voyait en eux que des adjuvants aux deux principaux.
} 
recherche d'un profit, mais des paysans qui produisent leur propre nourriture [...] L'indigence du réseau de transport interdit d'envoyer vers les grandes villes les produits périssables tels que le lait. "»

Examinons comment cette grille de lecture peut s'appliquer à l'Irlande, et comment la pomme de terre a pu y jouer un rôle comparable à celui du mil.

\section{Le système pomme de terre - lait}

Il est sans doute inutile d'insister ici sur les qualités, y compris organoleptiques, de la pomme de terre. On retiendra simplement, sur le plan agricole d'abord, sa faible exigence en matière de sols (qui permit la mise en culture de terres jusque-là inutilisées), sa grande productivité (ordinairement comprise entre 12 et 15 tonnes à l'hectare), la facilité de sa culture (le travail préalable de la terre et la plantation se font presque en surface, à $15-20 \mathrm{~cm}$ de profondeur seulement, l'apport de compost et l'arrosage n'étant de surcroît guère nécessaire dans le contexte irlandais), le caractère autant précoce que prolongé de sa récolte (à partir de trois mois et jusqu'à six mois après la plantation). On n'oubliera pas son rôle dans la bonification des sols, aptes ensuite à porter une récolte de céréales, ni sa facilité à être cuisinée (un feu de bois et une marmite suffisent) puis mangée (point besoin de couverts).

Sur le plan diététique, la pomme de terre apporte d'abord environ 80 calories $^{19}$ pour 100 grammes, cette donnée étant variable en fonction de l'espèce et du mode de préparation (essentiellement la durée de sa cuisson). Ceci équivaut à 800 calories au kilo, ou encore 800000 calories par tonne. Même en ne retenant que l'hypothèse basse d'une productivité de 12 tonnes à l'hectare, ce ne sont pas moins de dix millions de calories qu'une telle surface peut supporter chaque année, soit une disponibilité quotidienne de près de 30000 calories. Dans la mesure où les besoins moyens d'un être humain sont d'environ 2500 calories, ceux d'un travailleur de force ne montant qu'à $3200-3500$ environ, on voit qu'un hectare peut nourrir douze personnes. Dans le contexte irlandais, cela revient à dire qu'un acre de pommes de terre (un demi-hectare environ) suffit à nourrir une famille, et même son porc en sus ! Les consommations rapportées de cinq à six kilos par personne et par jour dépassent donc largement les seuls besoins, et ont correspondu de surcroît au passage à deux vrais repas par jour. C'est la réalité de cette abondance que L.A. Clarkson et E. Margaret Crawford ont mis en exergue dans leur ouvrage Feast and Famine: Food and Nutrition in Ireland 1500-1920 20 :

We entitled our book Feast and Famine to emphasise the point that there were many famine-free years and that in the years between famines there was enough food in Ireland to satisfy everybody. The word 'feast' is meant to convey the idea of a plenitude, even of an

\footnotetext{
${ }^{19}$ L'unité de base est la calorie, mais elle n'est guère utilisée car trop petite. On emploie de fait toujours son multiple, le kilocalorie, parfois également appelée " grande calorie ». Mais, presque toujours, on se contente de désigner ce dernier, de façon impropre, par le seul mot « calorie ».

${ }^{20}$ Oxford: University Press, 2001.
} 
abundance. This is not a notion that fits comfortably into the conventional story. Yet for much of her history, people in Ireland had plenty to eat. The rich ate more lavishly than the poor, but so did they everywhere. Over-indulgence was not a sin peculiar to the Irish upper classes.

Cette richesse nutritive de la pomme de terre est la conséquence de sa haute teneur $(19 \%)$ en glucides, ou sucres, qui l'apparente aux féculents, groupe qui rassemble les légumineuses (ex : les lentilles, petits pois, pois chiches, haricots secs et fèves), les céréales et le riz. Il est ici fondamental de noter qu'il s'agit de plus de sucres complexes, dits encore lents, qui sont métabolisés lentement, contrairement aux sucres rapides qui inondent rapidement le corps, lui apportant un véritable coup de fouet énergétique, immédiat mais sans durée. La pomme de terre est également riche en vitamines (notamment B1, B2, B6 et C, qui sont parmi les plus importantes) comme en minéraux (magnésium, potassium et fer). Elle ne contient certes que $2 \%$ environ de protides (ou protéines), mais on trouve parmi eux la quasi-totalité des acides aminés essentiels et des glycoprotéines dont l'être humain a besoin. Enfin sa richesse en eau (près de $80 \%$ de son poids) et sa quasi absence de graisse (ou lipide), à peine $0,1 \%$, ont font un aliment parfaitement diététique.

On note encore la supériorité de la pomme de terre sur le mil, car cet aliment ne présente presque aucune des qualités nutritionnelles de cette dernière, même s'il lui est comparable sur le plan agronomique ${ }^{21}$. De fait, à l'échelle du globe, tous les territoires ruraux qui en ont fait, faute de mieux, le fondement de leur subsistance, et notamment une large part de la Méso-Amérique, sont en sous-alimentation chronique. Il n'y a qu'une exception : précisément le Nordeste brésilien ! C'est que ce dernier complète le mil, un peu par la patate douce, ce qui n'est pas sans intérêt vu d'Irlande, et surtout par le lait. Ce produit est en effet non seulement produit localement en grande quantité, par les chèvres notamment mais, ne pouvant être ni stocké ni exporté vers les marchés urbains, trop distants et mal reliés par des routes médiocres, il est consommé localement. Selon de Castro encore : « le lait n'est nulle part, y compris en des régions bien plus riches, autant accessible à tous ».

Cette grande disponibilité du lait, y compris et surtout pour les plus pauvres, était une autre caractéristique de l'Irlande du premier $\mathrm{XIX}^{\mathrm{e}}$ siècle. Elle contribua largement à la bonne alimentation des enfants au cours de leurs premiers mois de vie et donc à maintenir la mortalité infantile à un niveau certes élevé, mais inférieur à ce que la pauvreté de leurs familles aurait dû impliquer. Dès que l'enfant pouvait l'ingérer, une purée de pomme de terre bien lactée remplaçait le lait seul, avec les mêmes effets positifs sur la santé. Par ailleurs, cette abondance du lait d'origine animale rendait moins nécessaire l'allaitement maternel, ce qui limita donc le rôle de frein à la fécondabilité féminine de ce dernier ${ }^{22}$. A une époque où le mariage était

${ }^{21}$ Il apporte la seule base calorique, mais ni les vitamines, ni les sels minéraux, ni les protéines qui sont nécessaires à l'être humain.

Après l'accouchement, le taux sanguin de prolactine (une hormone produite par l'hypophyse) augmente considérablement, ce qui déclenche chez la jeune mère la montée laiteuse ou lactogenèse. En cas d'allaitement, la succion du mamelon par le nouveau-né déclenche un réflexe neuro-hormonal qui maintient à un niveau élevé cette production de 
quasi systématique, et contracté plutôt jeune de surcroît (un peu après l'âge de vingt ans en règle, même si ce point est disputé), ce produit contribuait tant à la natalité générale qu'à la limitation de la mortalité infantile, et constituait ainsi un élément fondamental de l'étonnante croissance démographique de l'Irlande d'alors.

La seule véritable faiblesse intrinsèque de ce système pomme de terre - lait, outre bien sûr la survenue aléatoire de mauvaises récoltes pour la première, résidait dans sa vulnérabilité à la soudure, c'est-à-dire à la période où les réserves de l'année précédente étaient épuisées alors que la récolte de l'année n'était pas encore prête à être ramassée. Les Irlandais avaient alors recours à deux produits de substitution, en règle disponibles : les flocons d'avoine $\mathrm{d}$ 'abord, remarquablement adaptés au climat et au sol de l'Irlande, et qui avaient constitué la base alimentaire du pays avant d'être détrônés par la pomme de terre précisément. Les harengs, de façon plus marginale, contribuaient également à cette soudure.

Il n'y avait pas en revanche de parade à une mauvaise récolte de pommes de terre, car ce produit ne se conserve pas et les Irlandais qui en dépendaient ne disposaient d'aucune réserve alimentaire ou monétaire de substitution ${ }^{23}$. Leur unique forme de stockage résidait dans le porc de la famille, si elle en possédait (encore) un bien sûr. Ce porc n'était pas destiné à être mangé par ceux qui l'avaient élevé, mais à être vendu au marché, l'argent recueilli servant aux rares dépenses, à commencer par le loyer, qui exigeait un paiement en numéraire. La vente anticipée de l'animal en cas d'urgence représentait la seule possibilité de gagner du temps, mais en engageant l'avenir.

Plus grave encore fut l'érosion progressive de ce système tout au long du premier $\mathrm{XIX}^{\mathrm{e}}$ siècle $^{24}$, notamment en raison de la diffusion malheureuse de la variété lumper. Cette variété, originaire d'Écosse, fut importée vers 1810 en raison de ses excellents rendements et de sa résistance supposée aux maladies, malgré son goût médiocre qui en fit dans un premier temps un aliment pour le seul bétail. Mais ceci entraîna la volonté d'en planter partout ou presque, y compris sur les parcelles dédiées aux céréales et même celles abandonnées jusque-là aux chèvres ${ }^{25}$ - mais qui étaient aussi sources de lait! La mono-alimentation qui en résulta fut bien moins satisfaisante, car à la fois moins variée, moins goûteuse et moins équilibrée en raison de la baisse drastique de la consommation de lait. A l'heure du mildiou auquel elle se révéla fort sensible, cette dépendance à un produit unique devint l'un des facteurs aggravants de la Famine. Venons-en maintenant à cette dernière, en commençant par ceux des effets de la sous-alimentation que l'on peut qualifier de directs.

prolactine, et donc celle du lait. Mais ce taux élevé de prolactine dans le sang a également un effet inhibiteur sur la fonction ovarienne, et donc sur la fécondabilité de la mère.

${ }^{23}$ On citera encore ici Josué de Castro : "La cause ultime de la famine du Nordeste n'est pas la sécheresse, mais la pauvreté. Les gens n'ont pas les moyens de faire des stocks lorsque des surplus de nourriture sont disponibles. Dépourvus tant de réserve que de capacité d'achat, ils sont sans défense et tombent quand le fléau les frappe. "

${ }^{24}$ Pour une étude spécifique de cette période de changement, on pourra se reporter à l'ouvrage de Gearoid Ó TUATHAIGH, Ireland Before The Famine 1798-1848, publié en 1972 chez Gill et Macmillan.

${ }^{25}$ On pourra se référer, pour ce point, à l'excellent Atlas of the Irish Rural Landscape, publié en 1997 par les Presses Universitaires de Cork, et notamment à ses pages 85 à 89. 


\section{Mourir de faim stricto sensu}

Chez l'enfant tout d'abord, on distingue deux formes de malnutrition : le marasme nutritionnel, caractérisé par une double insuffisance en calories et en protéines, et le kwashiorkor, où la carence protidique est au premier plan. Le marasme concerne le très jeune enfant, de moins d'un an en règle, que sa mère ne peut soudain plus allaiter, soit parce qu'elle vient de mourir ou qu'elle est devenue globalement trop faible, soit - cas heureusement plus fréquent - parce que sa lactation s'est arrêtée. Ce processus physiologique est en effet délicat, et s'interrompt facilement si la mère est elle-même mal nourrie ou frappée par une infection intercurrente. Après une telle interruption le très jeune enfant, faute d'alimentation de substitution en quantité suffisante, commence à consommer ses propres tissus pour y trouver la source énergétique dont il a immédiatement besoin. La mort surviendra en quelques semaines, plus rarement en quelques mois, à la première infection ou par déshydratation aiguë. Lorsque cet enfant n'a pas atteint son premier anniversaire, on parlera à son sujet de mortalité infantile.

Si l'on peut présenter ce marasme nutritionnel comme une sorte d'accident, le kwashiorkor $^{26}$ est une pathologie bien plus banale. L'allaitement maternel est en effet parfaitement adapté aux besoins de l'enfant, sur le plan qualitatif du moins. Lorsqu'il vient à son terme, l'enfant passe brusquement à un régime dérivé de celui qui est servi aux adultes de sa famille, régime toujours moins satisfaisant pour lui, dans un premier temps du moins. En effet, même s'il reçoit une ration calorique qui est encore quantitativement suffisante, d'où la différence avec le marasme nutritionnel, cette ration se révèle bien moins riche en protéines que le lait maternel. Elle l'est tout particulièrement en protéines animales (viande, poisson, œuf et lait), qui ont le double avantage d'être les plus riches en amino-acides essentiels et les plus faciles à métaboliser par l'organisme. L'enfant débute alors, entre quelques semaines à quelques mois après le sevrage, une carence protéique d'autant plus nette que ses besoins en protéines avaient précisément commencé à devenir importants. Cette carence est bien connue car elle sévit largement, aujourd'hui encore, dans les pays en voie de développement, frappant les enfants au cours de leur deuxième et parfois même de leur troisième année de vie.

Ce contexte de sevrage délicat est donc important car il a permis de comprendre cette forme de malnutrition ${ }^{27}$. Toutefois le kwashiorkor peut survenir dans toutes les circonstances où l'enfant perd, de façon plus ou moins rapide, une alimentation qui était jusque-là globalement satisfaisante pour en recevoir une autre qui ne lui apporte pas assez de protéines. Tel est notamment le cas des épisodes de disette, et plus encore de famine, où le kwashiorkor peut frapper l'enfant jusqu'au delà de dix ans. Les enfants ainsi dénutris deviennent progressivement apathiques, perdent l'appétit (anorexie), tandis que le tableau clinique s'installe. Ce dernier

${ }^{26}$ Ce terme vient de la langue ashanti (Ghana), dans laquelle il veut dire : « maladie survenant chez un jeune éloigné de sa mère à l'occasion d'une nouvelle grossesse ». Il importe de noter que s'il connaît, logiquement, des synonymes dans d'autres langues vernaculaires, c'est lui qui a été retenu dans la Classification internationale des maladies (CIM) élaborée par l'Organisation mondiale de la santé (OMS). De même le terme «marasme nutritionnel» est un diagnostic de la CIM, indépendamment de l'usage courant du mot « marasme ».

${ }^{27}$ La description clinique n'a été réalisée qu'en 1933. 
combine quatre composantes à peu près constantes : des œdèmes (qui débutent aux pieds et aux chevilles), un retard de croissance (qui porte davantage sur la taille que sur le poids), une fonte musculaire (rendant difficile, puis impossible, tout effort physique) et des troubles psychomoteurs : l'enfant est apathique, irritable, parfois léthargique. Diarrhée et anémie complètent volontiers ce tableau qui, sans réalimentation, évoluera constamment vers la mort. Par contre, le kwashiorkor guérit en règle lorsqu'il est traité par ré-alimentation prudente, en quelques semaines seulement de surcroît, ce qui en dit long sur les privations de la Grande Famine et sur l'absence de réponse adaptée.

Pour compléter cette brève typologie des formes cliniques de l'insuffisance alimentaire chez l'enfant, il importe de noter que, lors d'accidents exceptionnellement brutaux (guerres, cataclysmes, et précisément famines), l'insuffisance de la ration calorique peut être telle que des enfants même âgés peuvent développer un véritable marasme nutritionnel, alors même que leurs besoins énergétiques sont très modérés, proportionnellement très inférieurs à ceux $\mathrm{du}$ nourrisson. Ceci fut notamment bien étudié pendant la guerre du Biafra (19671970). Dans ce cas, la rapidité de l'évolution, la fréquence des infections intercurrentes et les difficultés de la ré-alimentation engagent très rapidement le pronostic vital. Il est enfin possible, et ce fut probablement largement le cas à grande échelle pendant la Grande Famine, qu'une note de marasme soit venue se surajouter au kwashiorkor que l'on peut sans trop de risque qualifier de généralisé chez l'enfant, majorant ainsi le caractère péjoratif de la sous-nutrition.

Chez l'adolescent et l'adulte, dont les capacités de résistance sont supérieures, la baisse de la ration alimentaire se traduit par de nombreux mécanismes adaptatifs qui se mettent en place spontanément. Ainsi la destruction naturelle des protides du corps (catabolisme protéique) diminue, ce qui permet de les économiser et surtout de les faire transformer par le foie en sucre (néoglucogenèse), sucre qui est le combustible de la machine humaine. La néoglucogenèse en produit également à partir des réserves de graisse. Dans le même temps, le métabolisme se ralentit et les besoins énergétiques diminuent. La convergence de ces mécanismes, outrageusement simplifiés faut-il le dire, permet de gagner du temps, mais ce d'emblée au prix d'une baisse des capacités physiques, de travail notamment. Ces mécanismes de compensation finissent enfin par s'épuiser, faute de ressources, ce qui conduit à la mort. Mais, bien souvent, les protéines qui sont à la base de la défense immunitaire avaient déjà été trop réduites pour pouvoir assurer correctement leur fonction et une infection aura déjà frappé, et le plus souvent tué.

\section{Mourir d'une infection}

La nosographie des infections touchant une population en période de crise, telle une famine qui dura six ans, est assez complexe mais on peut, comme pour la malnutrition stricto sensu, en fixer à grands traits le cadre. Il faut d'abord commencer par saisir que, d'une manière générale et hors de tout contexte de calamité, l'être humain vit dans un monde rempli d'agents pathogènes et que l'infection lui est très banale. Ces agents peuvent être présentés - et classés - de multiples façons : le biologiste s'intéressera à leur nature (bactéries, virus, parasites ou champignons), le clinicien à leur gravité (bénigne, potentiellement grave chez le 
sujet affaibli ou encore redoutable pour tous), l'agent de santé publique enfin à leur mode de propagation (par l'eau ou hydrique, par l'air ou aérienne, par contact interhumain - direct ou indirect, via un réservoir animal, par piqûre d'insecte, etc.) et l'épidémiologiste à leur répartition.

Lors d'une catastrophe telle la Grande Famine, qui cumula durée et gravité, apparaissent deux facteurs qui se surajoutent à cette multiplicité pathogénique, mais qui vont aussi la simplifier en passant au tout premier plan. Le premier réside dans la baisse, puis éventuellement l'effondrement des défenses immunitaires. Dès lors la plupart des agents qui sont en règle sans gravité deviennent potentiellement létaux, et comprendre leur nature, leur mode d'action ou leur gravité potentielle n'a plus guère d'intérêt. Le second facteur réside dans l'apparition, en fait la réapparition, des grands pathogènes de l'humanité tels que le typhus, le choléra ou la fièvre récurrente. Ces derniers conjuguent une grande capacité de diffusion dans les groupes humains et une non moins grande capacité à tuer, y compris le sujet en bonne santé qui se trouverait là! A cela s'ajoute, bien sûr, y compris en notre époque postmoderne bien dotée en antibiotiques, le frisson que leur seul nom suffit à évoquer.

Une ultime simplification réside dans notre méconnaissance de ce qui a véritablement tué les Irlandais pendant la Famine. Cette méconnaissance se joue à deux niveaux, statistique et clinique. Statistiquement d'abord, si les recensements de 1841 et 1851 ont été, de l'avis des démographes ultérieurs, plutôt remarquables, ils ne contiennent guère de données fiables concernant la mortalité. La cinquième partie du recensement de 1851, publiée en 1856, s'intitule certes «Tableaux de la mortalitée ${ }^{28}$ ", et se risque même à tenter des quantifications, mais ces dernières sont extrêmement douteuses, ne serait-ce qu'en raison du nombre de décès méconnus (le quart de l'ensemble). Cormac Ó Gráda, déjà cité, a pu écrire à ce sujet dans le chapitre Mortality and the Great Irish Famine de l'Atlas de la Grande Famine, également déjà cité (cf. note 8 ) :

The data are seriously flawed, which probably explains why scholars have tended to shun them in the past. They report a total of 985,000 deaths between 1841 and 1851, or only slightly more than half the probable during the decade. The problem is that the census relied mainly on information given by survivors, but that would have meant the automatic exclusion of deaths in households that had disappeared in the interim.

Mais de telles insuffisances statistiques ont, au moins, le mérite d'être repérables, et donc de déclencher la prudence. En revanche, la seconde cause de notre méconnaissance des causes de mortalité pendant la Famine est plus insidieuse, ce qui impose de commencer par affirmer son existence. Il s'agit de l'état encore très médiocre des connaissances médicales de l'époque. Ceci dépasse largement la seule insuffisance thérapeutique, elle supposée connue, le premier antibiotique

${ }^{28}$ The Census of Ireland for the Year 1851, Part V, Tables of Deaths, Containing the Report, Tables of Pestilences, and Analysis of the Tables of Deaths, Dublin: Stationery Office, 1856. 
n'ayant - on le sait - été découvert qu'en $1929^{29}$. Ce qui est en jeu ici est la méconnaissance quasi complète à cette époque de la cause de la plupart des maladies. Pour en revenir brièvement et une dernière fois à la malnutrition, même l'éditeur des Tables de Mortalité que l'on vient de citer, l'éminent chirurgien irlandais William Wilde, qui couronna sa carrière en devenant le rédacteur en chef du Dublin Journal of Medical Science, précisément à partir de 1845, puis également comme «l'Ophtalmologiste ordinaire » de la reine Victoria, en distingua mal les diverses entités cliniques que nous avons rapidement précisées, tout simplement par ce qu'elles n'étaient pas encore bien déterminées ${ }^{30}$. On peut, peut-être, tout de même se souvenir de lui comme le père d'Oscar Wilde ${ }^{31} \ldots$ Les infections étaient, pour leur part, encore plus mal connues. Ainsi le choléra et la peste noire étaient-ils attribués à des «miasmes». Ce ne fut qu'en 1854 que, lors de l'épidémie qui ravagea le quartier de Soho à Londres en 1854, un certain John Snow eut l'idée de pointer sur un plan les domiciles des personnes contaminées. Il découvrit ainsi que cette épidémie était centrée sur une pompe d'eau publique, et mit en évidence la contamination par le biais de l'eau. La bactérie responsable, Vibrio cholerae, n'allait être identifiée par l'allemand Robert Koch qu'en 1883, mais dès la découverte de Snow on comprit que l'accès à une eau potable non souillée par des déjections constituait une prophylaxie efficace. Cette compréhension arriva un peu trop tard pour être utilisée dans l'Irlande de la Grande Famine.

Dans le cas du typhus, le mode de transmission (par le pou du corps) n'a été élucidé qu'en 1906 et l'isolement du germe en cause (Rickettsia prowazeki) réalisé en 1910. Quant à un traitement efficace par cyclines, il n'est disponible que depuis 1950. Il est toutefois probable que la connaissance de l'agent pathogène, et de son mode de transmission, n'aurait guère changé l'impact du typhus pendant la Grande Famine. En effet tous les facteurs de prolifération du pou (dénutrition, manque d'hygiène et promiscuité) y étaient présents sans que l'on puisse lutter contre eux. Reste que la méconnaissance clinique de cette affection a dû contribuer d'un côté à la méconnaître, alors qu'elle était bien présente, et de l'autre à la diagnostiquer par erreur, d'où des données statistiques à peu près sans intérêt. L'un de ses signes cardinaux, qui a donné son nom à la maladie, est le tuphos, qui consiste en un état d'hébétude et de stupeur. Ce tuphos ne se distingue guère de l'accablement général qui frappe une population dénutrie et sans espoir, d'où une source massive de diagnostics par excès. A l'inverse le décès par typhus survient en règle assez précocement (dès le $15^{\mathrm{e}}$ jour de la phase d'état) et brusquement, et il est facile de manquer la cause de la mort.

\footnotetext{
${ }^{29}$ Alexander Fleming découvrit la pénicilline G notamment grâce à la contamination fortuite de ses cultures par le champignon sur lequel travaillait son voisin de paillasse, un Irlandais du nom de Charles J. Latouche.

${ }^{30}$ Il distingua notamment (i) la mort de faim stricto sensu (starvation), qui peut correspondre à de multiples réalités cliniques sans que l'on puisse en savoir davantage, l'œdème de la faim (hunger oedema), qui rassemblait sans doute tant les kwashiorkors dont l'œdème était au premier plan que les autres causes d'œdèmes (hydropisie, insuffisances cardiaques, dysthyroïdie, etc.) et (iii) le marasme, lui déjà quelque peu connu mais dont Wilde n'imaginait pas qu'il puisse frapper un autre que le très jeune enfant.

${ }^{31}$ Ainsi que par le fait qu'il a eu l'occasion de soigner le père de George Bernard Shaw.
} 
Enfin, on sait aujourd'hui que le typhus est une famille de maladies, causées par des germes voisins mais néanmoins distinctes. Les éléments précédents concernent le seul Typhus exanthématique (ou Typhus historique), mais il est vraisemblable que d'autres typhus aient touché l'Irlande pendant la Grande Famine. Par ailleurs et comme pour complexifier encore plus les choses, on sait qu'une autre maladie infectieuse, très semblable, était présente: la fièvre récurrente. Cette dernière est également transmise par le pou du corps, donne également une fièvre élevée et peut évoluer rapidement vers une crise létale. Mais tout ceci n'est connu que depuis le début du $\mathrm{XX}^{\mathrm{e}}$, et dans l'Irlande de la Famine nombre de ces fièvres ont pu être prises pour des cas de typhus, et vice-versa.

Si ces faits étaient inconnus des médecins de l'époque, on imagine la qualité des diagnostics post-mortem posés par agents préposés à cette tâche et, donc, la faible qualité (déjà notée plus haut) des statistiques. Citons-les néanmoins, puisqu'elles sont susceptibles de donner un ordre de grandeur à l'échelle de l'Irlande entière : $29,2 \%$ de décès par "fièvre», $24,9 \%$ par pathologie digestive, généralement dans un contexte de diarrhée, $24,3 \%$ de décès sans cause connue, $10,0 \%$ par cachexie de malnutrition, $6,8 \%$ par choléra et enfin $4,8 \%$ par pathologie respiratoire.

Il apparaît donc, à ce stade de la réflexion, que le faible niveau des connaissances médicales de l'époque, notamment en nutrition et en pathologie infectieuse, rend totalement illusoire toute explicitation des causes de la mortalité pendant la Grande Famine, et ce indépendamment de la qualité (ou de la non qualité) du recueil statistique des données. On peut affiner cette affirmation en notant que, dans toutes les pathologies que l'on vient d'examiner, ce faible niveau correspondait, selon les cas, soit à des connaissances imparfaites mais dont le socle était déjà présent pour le futur (cas du marasme nutritionnel), soit à une absence totale de connaissance de la réalité (cas du choléra). Mais ceci n'épuise pas les possibilités, et il nous reste à examiner pour finir un troisième et ultime degré d'ignorance, celui des tentatives de compréhension qui allaient se révéler complètement erronées à terme et, de plus, parfois dangereuses sur le moment.

\section{Une ignorance mortelle}

Commençons par ce que les médecins de l'époque appelaient «la Fièvre». Connue comme concept depuis le XII ${ }^{\mathrm{e}}$ siècle, crainte comme le pire fléau de l'île, elle était attribuée, selon les cas, à la pauvreté, au manque de nourriture, à l'habitat insalubre, mais aussi à la proximité d'avec le porc de la famille ou à l'alcoolisme. En 1848 encore, un médecin du comté d'Offaly (alors appelé comté du Roi) attribua le début d'une épidémie de cette "fièvre » à une aurore boréale... Sa fréquence particulièrement élevée pendant la Famine fit développer le concept de « fièvre de la faim », dont on pense aujourd'hui qu'il regroupe des réalités cliniques diverses, qu'il serait bien sûr illusoire d'espérer comprendre a posteriori. Mais avant même 1845, la crainte de « la fièvre » et de sa contagiosité avait conduit les autorités à construire des établissements spécialisés, les «hôpitaux de la fièvre", dont certains étaient régis - et financés - par les autorités en charge de la Poor Law. Il est estimé qu'une centaine environ de ces hôpitaux existaient à la veille de la Famine, et que plus de 350 autres furent établis au fur et à mesure que l'urgence s'étendait. Si l'idée de 
protéger la population encore indemne en assurant l'isolement des infectés est de fait une excellente idée, le mélange des patients atteints d'affections différentes ne pouvait que faciliter la dissémination de l'ensemble des pathologies présentes, et donc d'accroître encore la mortalité dans ces Fever Hospitals. Par ailleurs, leur localisation rarement isolée mais plutôt au centre des bourgs fut un facteur majeur de contagiosité de la population générale.

Semblablement, les très fréquents épisodes de diarrhées abondantes étaient en règle qualifié de «dysenterie », alors que seule une petite partie d'entre eux sans doute étaient véritablement des dysenteries au sens précis où on emploie ce terme aujourd'hui, à savoir une infection digestive par une bactérie du genre shigella. Dans la mesure où presque toute la pathologie infectieuse - et nutritionnelle - peut s'accompagner d'épisodes diarrhéiques, on conçoit qu'un tel étiquetage large ne permette en aucune façon d'espérer reconstituer la réalité. Il présentait de plus une connotation fort péjorative, Cromwell ayant paraît-il qualifié la dysenterie de « maladie de l'Irlande »!

On peut retirer deux enseignements de cet emploi générique des mots «fièvre » et «dysenterie ». Le premier, fondamental, est qu'un quart de siècle seulement avant la révolution pasteurienne, que l'on peut considérer ouverte par la publication en 1878 de La théorie des germes et ses applications à la médecine et à la chirurgie, le savoir médical était encore largement empirique. Il importe donc de ne pas faire d'anachronisme dans l'évaluation de la prise en charge de la Grande Famine. Le second, quelque peu symétrique, consiste à comprendre que le corps médical irlandais, qui paya un lourd tribut aux hôpitaux de la fièvre (et n'apprécia guère de n'y être payé que cinq shillings par jour pour voir parfois plus de deux cent malades), utilisa à l'extrême son sens de l'observation pour essayer de comprendre les diverses formes de fièvre et de diarrhée, même si ceci ne put conduire à des avancées scientifiques immédiates. Par exemple certains savaient parfaitement distinguer, sans les nommer correctement bien sûr, le typhus exanthématique et la fièvre récurrente, comme des entités nosographiques voisines mais distinctes. La Grande Famine s'est déroulée un peu avant la révolution pastorienne, mais la médecine du temps était déjà bien loin de son ancêtre médiévale.

Ses insuffisances étaient toutefois bien connues des autorités politiques, au Royaume-Uni comme ailleurs, alors que la chimie organique brillait de tous ses feux depuis le début de siècle. Ceci explique que la Commission chargée par Peel de découvrir la cause de la mauvaise récolte s'obstina à ignorer l'hypothèse d'un champignon pathogène et chercha en vain une cause chimique. Le fait est bien connu, mais que la Commission, contrairement à ses préjugés de départ, finit par conclure que chimiquement la pomme de terre était finalement un aliment très remarquable, voire l'un des meilleurs, l'est peut-être moins ! Il n'est dès lors guère surprenant que le Central Board of Health, établi en mars 1846, ait consacré une large part de son énergie à chercher le mode de préparation de la pomme de terre qui soit le plus nutritif possible. On se trouve là, ce me semble, devant une parfaite combinaison d'insuffisance scientifique et de préjugé politique. 


\section{Conclusion}

Ce panorama des causes de la mortalité pendant la Grande Famine n'ayant aucune prétention à l'exhaustivité, on n'y aura donc trouvé aucune mention d'affections telles que la variole ou le scorbut. Son objectif était, d'abord, de rendre intelligible une situation qui est de fait complexe, et que souvent l'on n'ose examiner du fait de sa participation au champ biomédical. Au terme de ce parcours, il me semble que l'on peut tenir les six propositions suivantes :

1. Sauf exception les Irlandais, y compris les plus pauvres, étaient bien, voire très bien alimentés à la veille de la Grande Famine.

2. Ceci résultait de leur régime fondé sur le couple lait/pomme de terre, $\ldots$

3. ... mais la diffusion de la variété lumper fragilisa progressivement ce système à partir de 1810, indépendamment des changements sociétaux et de la pression démographique bien connus par ailleurs. faim.

4. La mortalité globale des enfants fut effroyable, surtout par impact direct de la

5. Celle des adultes est quantitativement et qualitativement mal connue, et il est illusoire tant d'espérer la chiffrer que la ventiler par cause. Il apparaît même déraisonnable de se risquer à une tentative de répartition grossière entre les morts de faim stricto sensu et les morts par infection.

6. Ceci résulte de l'insuffisance combinée de l'appareil statistique et du savoir médical de l'époque. 